\title{
Pulmonary epithelioid hemangioendothelioma presenting with vertebral metastases: a case report
}

Angela Sardaro ${ }^{1 *}$, Lilia Bardoscia ${ }^{1}$, Maria Fonte Petruzzelli ${ }^{1}$, Anna Nikolaou ${ }^{1}$, Beatrice Detti ${ }^{2}$ and Giuseppe Angelelli ${ }^{1}$

\begin{abstract}
Introduction: Epithelioid hemangioendothelioma is a rare vascular tumor that has an epithelioid and histiocytoid appearance, originates from vascular endothelial or pre-endothelial cells and comprises less than $1 \%$ of all vascular tumors. It was described for the first time in 1975 as pulmonary epithelioid hemangioendothelioma, because initially it was believed to be an aggressive form of bronchoalveolar cell carcinoma with a remarkable propensity to invade adjacent blood vessels and small airways. Only a few cases have been reported in the literature to date. Tumor cells expressing Fli-1 and CD31 have been identified as relatively specific endothelial markers. Epithelioid hemangioendothelioma may affect multiple organs and may vary considerably in its clinical and radiological presentation. More than $50 \%$ to $76 \%$ of pulmonary epithelioid hemangioendothelioma patients are asymptomatic. They are usually incidentally diagnosed on the basis of abnormal chest radiography during routine physical examinations. Hematologic and gastrointestinal disorders and weakness or numbness may also be observed, in addition to respiratory symptoms, in cases of disseminated pulmonary epithelioid hemangioendothelioma. Pain and swelling, pathological fractures, spine compression or paresthesia, loss of muscular strength and paraplegia may be present when bone metastases occur. Because of the rarity of this disease, there is no standard for treatment.

Case presentation: A 46-year-old Caucasian woman presented to our institution in November 2009 with metastases of pulmonary epithelioid hemangioendothelioma from the $L 3$ and $L 4$ vertebrae. A course of radiotherapy at a dosage of 3,000cGy delivered in individual doses of 200cGy/day for 5 days/wk to the $L 3$ and $L 4$ vertebrae led to the disappearance of the patient's lumbar pain without any detectable side effects. Percussion of the patient's vertebral spine was negative, and no radiological progression of bone disease was found at her 1-year follow-up examination.

Conclusion: Since epithelioid hemangioendothelioma was first correctly defined, several research groups have reported their experiences with epithelioid hemangioendothelioma irradiation. Further studies are needed to establish a standard radiation dose to be used for such a complex and extremely rare disease. In our present case, a radiotherapy dosage of 3,000cGy delivered in individual doses 200cGy/day for 5 days/wk allowed us to reach our goals: local pain control with good tolerance and better quality of life by the 1-year follow-up examination.
\end{abstract}

Keywords: Bone metastases, Endothelial markers, Epithelioid hemangioendothelioma, Radiation therapy, Vascular tumor

\footnotetext{
* Correspondence: angela.sardaro@uniba.it

${ }^{1}$ Azienda Ospedaliero-Universitaria Policlinico di Bari, Dipartimento

Interdisciplinare di Medicina, Sezione di Diagnostica per Immagini e

Radioterapia, Università degli Studi di Bari "Aldo Moro", Piazza Giulio Cesare

11, 70124 Bari, Italia

Full list of author information is available at the end of the article
}

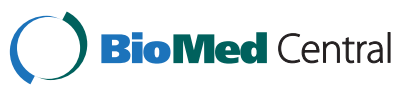

(c) 2014 Sardaro et al.; licensee BioMed Central Ltd. This is an Open Access article distributed under the terms of the Creative Commons Attribution License (http://creativecommons.org/licenses/by/4.0), which permits unrestricted use, distribution, and reproduction in any medium, provided the original work is properly credited. The Creative Commons Public Domain Dedication waiver (http://creativecommons.org/publicdomain/zero/1.0/) applies to the data made available in this article, unless otherwise stated. 


\section{Introduction}

Representing less than $1 \%$ of all vascular tumors, epithelioid hemangioendothelioma (EHE) is a rare vascular tumor with an epithelioid and histiocytoid appearance that originates from vascular endothelial or pre-endothelial cells. It was described for the first time in 1975 by Dail et al. as pulmonary epithelioid hemangioendothelioma (PEH). Initially, it was believed to be an aggressive form of bronchoalveolar cell carcinoma with a remarkable propensity to invade adjacent blood vessels and small airways; hence the name intra-vascular bronchioloalveolar tumor [1].

The term epithelioid hemangioendothelioma was introduced in 1982 by Weiss and Enzinger in describing a vascular tumor of bone and soft tissue with intermediate malignancy and features between hemangioma and angiosarcoma [2]. Weldon-Linne et al., by using electron microscopy, confirmed previous findings about these tumor cells that they derive from a lineage capable of differentiation along endothelial lines. By immunohistochemistry, they revealed diffuse cytoplasmic staining of the malignant cells with a factor VIII-related antigen, which clarified the endothelial lineage of these tumor cells [3].

The World Health Organization 2002 classification [4] describes such tumors as lesions that fall into the category of locally aggressive tumors with metastatic potential. Therefore, some authors began to believe the hypothesis that EHE does not represent a distinct entity, but rather is an intermediate state of endothelial dedifferentiation with a highly variable and unpredictable prognosis [5].

Herein we present the case of a patient with extensive dissemination and serious bone damage due to $\mathrm{PEH}$ who benefited from radiation therapy (RT) for pain and control of local bone recurrence.

\section{Case presentation}

A 46-year-old Caucasian woman presented to our institution in November 2009 with L3 and L4 vertebrae metastases of PEH. Previous thoracopulmonary investigations had revealed multiple disseminated pleuropulmonary nodules followed by pulmonary wedge resection, confirming the typical clinical PEH immunoreactivity for CD31, factor VIII, Fli-1 and vimentin. She had also undergone lumbar computed tomography $(\mathrm{CT})$, which showed structural changes in the third and fourth lumbar vertebrae (L3-L4) combined with a partially collapsed L4 and L3-L4 disc protrusion. Her abdominal CT scan had shown a small, common iliac vein thrombosis and a small caval thrombosis immediately above the iliac venous confluence. All of these findings together led to initiation of therapy with polyethylene glycol and interferon $\alpha$ at a dosage of $1 \mu \mathrm{g}$ to $2 \mu \mathrm{g}$ once every 7 days. A CT scan performed 4 months after initiation of treatment revealed stable lung disease but new hypodense splenic lesions. A lumbar spine magnetic resonance imaging scan showed exacerbation of bone disease with worsening L3 and L4 collapse and further L4-L5 disc protrusion. Therefore, she underwent kyphoplasty. The bone biopsy performed during the operation confirmed the diagnosis of $\mathrm{L} 4$ vertebral body EHE.

When the patient presented to our institution, she complained of back pain that did not go away after taking common anti-inflammatory drugs and treatment with lumbar vertebrae acupressure. The pain was absent in other areas. On the basis of the scarce available literature on patients with this presentation, we delivered symptomatic RT into the L3-L5 vertebral tract using a 3,000cGy total target dose in 200cGy daily fractions 5 days/wk. At the end of the treatment, the patient's lumbar pain had disappeared and she experienced no detectable side effects. In 2010, we observed negative percussion of the vertebral spine and no radiological progression of bone disease. The patient underwent ifosfamide and epirubicin chemotherapy and surgical removal of EHE spleen lesions soon after her follow-up examination at our institution. She died in October 2010 as a result of PEH progression.

\section{Discussion}

After Dail et al. first identified PEH in 1975 [1], in 1982 Weiss and Enzinger used the term epithelioid hemangioendothelioma to describe a vascular bone and soft-tissue tumor with intermediate malignancy between hemangioma and angiosarcoma [2]. Weldon-Linne et al. discovered a factor VIII-related antigen in malignant cells, confirming their endothelial origin [3]. The median age of onset is 36 years (range, 20 to 60 years) [6]. The most common presentations are liver alone (21\%), liver plus lung (18\%), lung alone (12\%) and bone alone (14\%) [7]. Approximately 50\% to $76 \%$ of patients are asymptomatic. Some present with chest pain, pleuritic pain, cough, dyspnea, malaise [8], hemoptysis and anemia. Bone metastases cause pain and swelling in the affected area, pathological fractures [9], spine compression if the lesions arise in vertebrae, which result in paresthesia, loss of muscular strength and paraplegia [10]. Hematologic and gastrointestinal disease, as well as weakness or numbness, may also occur because of EHE dissemination [7]. Radiologically, $\mathrm{PEH}$ consists of multiple bilateral perivascular nodular opacities with a diameter $\leq 1 \mathrm{~cm}$ [10], and sometimes present with lymph node metastases, pleural thickening or ground-glass opacities. Bone metastases appear as osteolytic lesions with homogeneous contrast enhancement, cortical disruption and soft-tissue swelling [9]. Histologically, the tumor has a micropolypoid growth, and tumor cells with cytoplasmic vacuoles occasionally contain erythrocytes [11] expressing Fli-1 and CD31 [12], which are relatively specific endothelial markers. The overall survival rate of EHE patients is better when the disease is localized (90\% at 1 year and $73 \%$ at 5 years) 
than when there is multi-organ progression $(53 \%$ at 1 year and $24 \%$ and 5 years) [7].

Because of the rarity of this disease, there is no standard for treatment. Curative resection achieves good outcomes. Usually, adjuvant RT is used to control residual disease for patients with localized EHE. Chemotherapy (interferon $2 \alpha$ or carboplatin plus etoposide) is the preferred therapy for patients with widespread disease, but the benefits of these drugs are unclear $[1,6,13]$.

Because of the radiobiological characteristics of PEH (slow growth), RT is ineffective. Good local control has been reported in a patient with exclusive EHE bone presentation treated by combining RT with bone surgery [14]. From the time EHE was correctly defined, several research groups have studied irradiation for EHE. A protocol of 4,000 cGy for 4 weeks [13], followed by a 3,000cGy course of RT to the spine after surgical removal of EHE of the vertebrae [15] has been described. In the study reported by Aquilina et al., one patient survived for 11 years, but the other patients experienced worsening conditions due to multiple hepatic and abdominal metastases. Authors of another report described adjuvant RT delivered at dose of 6,400cGy to treat axillary EHE, which resulted in the absence of lymph node metastases but widespread pleuropulmonary metastasis [6]. Local irradiation after bone EHE resection at 6,000cGy in 23 fractions for 43 days showed good tolerance, without regional or distant metastases or local recurrence at 6-, 12- and 24-month follow-up examinations [10]. A recently reported protocol of 33 radiation fractions totaling 5,940cGy was applied to treat residual mastoid EHE. The authors found that imaging performed 8 years after surgery and RT revealed neither recurrence nor any change in the patient's clinical status [16].

\section{Conclusion}

Further studies are needed to establish the standard radiation dose to be used for locoregional control of this complex and extremely rare disease. In our present case, a RT dosage of 3,000cGy delivered at 200cGy/day 5 days/wk allowed us to reach our goals: local pain control with good tolerance and better quality of life at the 1-year follow-up examination.

\section{Consent}

Written informed consent was obtained from the patient's next of kin for publication of this case report and any accompanying images. A copy of the written consent is available for review by the Editor-in-Chief of this journal.

\section{Abbreviations}

EHE: Epithelioid hemangioendothelioma; PEH: Pulmonary epithelioid hemangioendothelioma; RT: Radiation therapy (Radiotherapy).
Competing interests

The authors declare that they have no competing interests.

\section{Authors' contributions}

AS analyzed and interpreted the patient data regarding the physical, radiological and histological examinations, elaborated the radiation treatment plan and was a major contributor to the writing of the manuscript. $L B$ reviewed the available literature. MFP and AN assembled this report. BD and GA supervised the work. All authors read and approved the final manuscript.

\section{Author details}

${ }^{1}$ Azienda Ospedaliero-Universitaria Policlinico di Bari, Dipartimento Interdisciplinare di Medicina, Sezione di Diagnostica per Immagini e Radioterapia, Università degli Studi di Bari "Aldo Moro", Piazza Giulio Cesare 11, 70124 Bari, Italia. ${ }^{2}$ Radioterapia, Azienda Ospedaliero-Universitaria Careggi, Largo Brambilla 3, 50134 Firenze, Italia.

Received: 12 February 2014 Accepted: 7 April 2014

Published: 18 June 2014

\section{References}

1. Dail DH, Liebow AA, Gmelich JT, Friedman PJ, Miyai K, Myer W, Patterson SD, Hammar SP: Intravascular, bronchiolar, and alveolar tumor of the lung (IVBAT): an analysis of twenty cases of a peculiar sclerosing endothelial tumor. Cancer 1983, 51:452-464.

2. Weiss SW, Enzinger FM: Epithelioid hemangioendothelioma: a vascular tumor often mistaken for a carcinoma. Cancer 1982, 50:970-981.

3. Weldon-Linne CM, Victor TA, Christ ML, Fry WA: Angiogenic nature of the "intravascular bronchioloalveolar tumor" of the lung: an electron microscopic study. Arch Pathol Lab Med 1981, 105:174-179.

4. World Health Organization (WHO); International Association for Research on Cancer: In World Health Organization Classification of Tumours: Pathology and Genetics of Tumours of Soft Tissue and Bone. Edited by Fletcher CDM, Unni KK, Mertens F. Lyon, France: IARC Press; 2002. Available at http://www.iarc.fr/ en/publications/pdfs-online/pat-gen/bb5/BB5.pdf.

5. Liu Q, Miao J, Lian K, Huang L, Ding Z: Multicentric epithelioid hemangioendothelioma involving the same lower extremity: a case report and review of literature. Int J Med Sci 2011, 8:558-563.

6. Schattenberg T, Kam R, Klopp M, Herpel E, Schnabel PA, Mechtersheimer G, Dienemann H, Pfannschmidt J: Pulmonary epithelioid hemangioendothelioma: report of three cases. Surg Today 2008, 38:844-849.

7. Lau K, Massad M, Pollak C, Rubin C, Yeh J, Wang J, Edelman G, Yeh J, Prasad S, Weinberg G: Clinical patterns and outcome in epithelioid hemangioendothelioma with or without pulmonary involvement: insights from an internet registry in the study of a rare cancer. Chest 2011, 140:1312-1318.

8. Sicilian L, Warson F, Carrington CB, Hayes J, Gaensler EA: Intravascular bronchioloalveolar tumor (IV-BAT). Respiration 1983, 44:387-394.

9. Larochelle O, Périgny M, Lagacé R, Dion N, Giguère C: Epithelioid hemangioendothelioma of bone. Radiographics 2006, 26:265-270.

10. Gherman CD, Fodor D: Epithelioid hemangioendothelioma of the forearm with radius involvement: case report. Diagn Pathol 2011 6:120

11. Murali R, Zarka MA, Ocal IT, Tazelaar HD: Cytologic features of epithelioid hemangioendothelioma. Am J Clin Pathol 2011, 136:739-746

12. Gill R, O'Donnell RJ, Horvai A: Utility of immunohistochemistry for endothelial markers in distinguishing epithelioid hemangioendothelioma from carcinoma metastatic to bone. Arch Pathol Lab Med 2009, 133:967-972.

13. van Kasteren MEE, van der Wurff AAM, Palmen FMLHG, Dolman A, Miseré JFMM: Epithelioid haemangioendothelioma of the lung: clinical and pathological pitfalls. Eur Respir J 1995, 8:1616-1619.

14. Kabukçuoğlu F, Kabukçuoğlu Y, Livaoğlu A, Ozağari A, Armağan R, Kuzgun $\mathrm{U}$ : [Epithelioid hemangioendothelioma of bone] [in Turkish]. Acta Orthop Traumatol Turc 2006, 40:324-328. 
15. Aquilina K, Lim C, Kamel MH, Marks CJ, O'Sullivan MG, Keohane C:

Epithelioid hemangioendothelioma of the spine: report of two cases. J Neurosurg Spine 2005, 3:393-399.

16. Drazin D, Gandhi R, Slodkowska E, Boulos AS: Epithelioid

hemangioendothelioma of the mastoid: resection for recurrence and adjuvant radiation with 8-year followup. Case Rep Surg 2013, 2013:469201.

doi:10.1186/1752-1947-8-201

Cite this article as: Sardaro et al:: Pulmonary epithelioid

hemangioendothelioma presenting with vertebral metastases: a case report. Journal of Medical Case Reports 2014 8:201.

\section{Submit your next manuscript to BioMed Central and take full advantage of:}

- Convenient online submission

- Thorough peer review

- No space constraints or color figure charges

- Immediate publication on acceptance

- Inclusion in PubMed, CAS, Scopus and Google Scholar

- Research which is freely available for redistribution 